\title{
Force of Infection and Evolution of Lesions of Canine Tegumentary Leishmaniasis in Northwestern Argentina
}

\section{Jorge Diego Marco, Angel Marcelo Padilla, Patricio Diosque, Marisa Mariel Fernández*, Emilio Luis Malchiodi*, Miguel Angel Basombrío/ ${ }^{+}$}

Laboratorio de Patología Experimental, Facultad de Ciencias de la Salud, Universidad Nacional de Salta, Calle Buenos Aires 177, 4.400 Salta, Argentina *Instituto de Estudios de Inmunidad Humoral, Cátedra de Inmunología, Facultad de Farmacia y Bioquímica, Universidad de Buenos Aires, Buenos Aires, Argentina

A clinical-serological follow-up was carried out in a canine population in endemic foci of Leishmania braziliensis spread in northwestern Argentina. Each dog was studied in at least two visits, $309 \pm 15$ days $(X \pm S E)$ apart. Some initially healthy dogs $(n=52)$ developed seroconversion or lesions. The clinical evolution of the disease in dogs resembles in many aspects the human disease. Similarities include the long duration of most ulcers with occasional healing or appearance of new ones and the late appearance of erosive snout lesions in some animals. Yearly incidence rates of $22.7 \%$ for seroconversion and of $13.5 \%$ for disease were calculated as indicators of the force of infection by this parasite upon the canine population.

Key words: dog - tegumentary leishmaniasis - force of infection - follow-up - Argentina

Dogs are highly susceptible to the development of leishmaniotic ulcerative lesions in areas where Leishmania (Viannia) braziliensis is transmitted among human populations. The possible role of these animals as reservoirs and their availability as targets for field studies on vaccination and therapy, add a medical and epidemiological dimension to the veterinary problem. Many "cross sectional" surveys and very few "longitudinal", follow-up studies have been performed on canine tegumentary leishmaniasis (CTL). Using the former approach, several authors (Reithinger \& Davies 1999) have determined the pathologic, serologic and parasitologic features of CTL. Alternatively, follow-up studies of canine populations determining the risk of acquiring infection in non-infected animals, the evolution of recent infection, and the outcome of incipient or advanced disease, are quite relevant for vaccination

This work received financial support from Consejo Nacional de Investigaciones Científicas y Técnicas, Consejo de Investigación of the University of Salta, Universidad de Buenos Aires and Agencia Nacional de Promoción Científica y Tecnológica.

${ }^{+}$Corresponding author. Fax: 54-387-425.5333. E-mail: basombri@ciunsa.edu.ar

Received 31 July 2000

Accepted 13 December 2000 or therapy and have seldom been addressed in CTL. With this purpose, we have undertaken a "longitudinal" follow-up study of non-infected, recently infected and chronically infected dogs of a high-transmission region.

The work was undertaken at the departments of Orán and San Martín, province of Salta, Argentina. Humid forests abound in this area, where intensive agriculture has expanded in the last three decades. Many human cases of leishmaniotic cutaneous or mucocutaneous ulcers have been recorded in recent years. Most lesions occur in rural personnel working at the edge of deforestation fronts. Urban outbreaks in the cities of Tartagal and Pichanal where recorded in 1985 and 1994 (Sosa Estani et al. 1998).

The dogs studied lived in rural or periurban dwellings, selected for having had human patients in either the same house or in the close vicinity. Although we have so far been unable to characterize the species of Leishmania isolated from dogs, at least seven out of eight human Leishmania isolates from the same area were classified by different methods as belonging to the subgenus Viannia, braziliensis complex (Campanini et al. 1993, Cuba et al. 1996, Sinagra et al. 1997). Groups which have succeeded in the isolation of dog parasites in other areas of human transmission of $L$. (V.) braziliensis could show that most dog isolates were homologous to the human ones (Cuba et al. 1985, Aguilar et al. 1987). 
A serologic, clinical and parasitologic followup was carried out in three groups of animals: Group 1: apparently healthy dogs, confirmed as seronegative, $n=52$; Group 2: seropositive dogs, bearing ulcerative lesions $(\mathrm{n}=28)$ and Group 3: seropositive dogs without clinically detectable lesions $(n=3)$. Every dog was visited, examined and analyzed at least twice. The interval between the first and the last rounds of visits averaged 309 days (standard error: 15 days; range: $95-625$ days). The skin of each animal was examined in detail, paying special attention to ears and genitals.

Blood was drawn by venous puncture and the serum was kept frozen until tested with an ELISA assay (Malchiodi et al. 1994). A panel of antigens, including F45 of L. mexicana, and Ag163B6 of Trypanosoma cruzi allowed the distinction between Leishmania-infected (F45+, Ag163B6-) and T. cruzi infected dogs (Ag163B6+; Chiaramonte et al. 1999).

The criteria used to define lesions as "compatible with leishmaniasis" were: ulcerative character, long duration, and rounded, raised and indured edges. Lesions probably induced by trauma were not considered as due to Leishmania infection.

Material for Giemsa-stained smears was obtained mainly by either scratching the ulcer margin with a toothpick or by touch-printing fresh biopsied skin tissue.

Group 1 - Clinically, these dogs showed no lesions and had been completely seronegative in the first round of visits. In the subsequent rounds, 6/ $52(11.6 \%)$ had developed typical ulcerative lesions, characteristic of leishmaniasis and 10/52 $(19.2 \%)$ had seroconverted. Coincidence between appearance of lesions and seroconversion occurred in three dogs, seroconversion without apparent lesions occurred in seven and lesions without seroconversion in three.
Group 2 - This group had 28 dogs bearing lesions, all of them with positive serology in the first round. Half of these animals maintained their lesions without noticeable cure or aggravation during all the period of observation. In four animals (14.3\%) bearing multiple lesions in the first round, it was observed that the ear or snout lesions progressed while other lesions, mostly on furred skin, underwent cure (Table, "divergent evolution"). Seven dogs (25\%) of this group showed evident aggravation of their lesions, which increased either in size or number. Finally, in the remaining three dogs, $(10.7 \%)$ complete cure was observed (Table).

Group 3 - Of the three seropositive dogs without lesions in the first round, two developed no lesions and the remaining one developed a large lesion in the ear.

The interval between the first and last observation of each dog (309 \pm 15 days) was only a fraction of the lifetime of these animals. However, our observation period was enough to witness several primary infections, as attested by 10/52 cases of seroconversion, the appearance of new lesions in $7 / 58$ animals, the aggravation or persistence of lesions in 25/28 animals and apparent cures in a small proportion of them (3/28). The "divergent evolution" of multiple lesions, where some lesions in snout and ears aggravated and others (mostly in furred skin) improved, seems to be consistent with two stages in this disease. The first would consist of lesions occurring at the site of inoculation (Kirkpatrick et al. 1987). The second would involve the metastatic, progressive, erosive mucocutaneous nasal lesions, equivalent to the so-called "espundia" or "uta" in humans (WHO 1990). The fact that either lesions in the nasal or auricular cartilage were mostly found in elderly dogs, further supports this assumption. The average age of dogs

\section{TABLE}

The appearance and evolution of leishmaniotic lesions in seronegative (group 1) and seropositive (groups 2 and 3) dogs

\begin{tabular}{cllcr}
\hline Group $^{a}$ & First round & Evolution & Proportion & $\%$ \\
\hline 1- Seronegative, & Normal skin & Normal skin & $46 / 52$ & 81 \\
no lesion & Normal skin & Ulcer & $6 / 52$ & 19 \\
2 - Seropositive & Ulcer & Persistent ulcer & $14 / 28$ & 50 \\
with lesion & Multiple lesion & Divergent evolution & \\
& Ulcer & Aggravation & $4 / 28$ & 14 \\
& Ulcer & Cure & $7 / 28$ & 25 \\
3 - Seropositive, & Normal skin & No lesion & $3 / 28$ & 11 \\
no lesion & Normal skin & New lesion & $2 / 3$ & 66 \\
\hline
\end{tabular}

$a$ : serology in this column refers to the first round; $b$ : divergent evolution: some of the original lesions aggravated and other ones cured. 
with erosive snout or ear lesions $(\mathrm{n}=10)$ was $5.4 \pm$ 1.7 years whereas dogs with lesions in other sites $(n=48)$ were $3.6 \pm 2.2$ years old $(\mathrm{p}<0.014)$.

Given the rates of seroconversion/lesions and the average intervals between observations in our samples, a yearly incidence rate of $22.7 \%$ for seroconversion and of $13.5 \%$ for disease can be expected for normal dogs exposed under the same epidemiological conditions. Studies on the force of infection for canine visceral leishmaniasis in northern Brazil (Quinnell et al. 1997), based on estimates of per capita incidence rates and basic reproduction number, have shown an even stronger risk of infection, pointing to the high susceptibility of dogs to both types of leishmaniasis.

The serological and clinical survey of dogs owned by highly exposed human communities might thus provide a measurable and consistent parameter to evaluate therapeutic or preventive measures, including vaccination.

Both parasite load and time of exposure to vectors are main determinants of the ability of canine populations to disseminate the infection. In a previous report (Padilla et al. 1999) we have described the scarcity of parasites in dog lesions. Only 14 of 25 smears were positive and the load was much scantier than in human lesions of the same area. This raised doubts about the role of dogs as reservoirs. However, the present observations point again to the importance of dogs in this respect. Sand fly-mediated propagation of CTL is highly dependent on whether the insects feed on lesions as opposed to normal skin (Vexenat et al. 1986). The long periods during which dogs maintain open, exposed lesions, might counterbalance the scarcity of parasites to secure an efficient vectorial transmission.

\section{ACKNOWLEDGEMENTS}

To Maria Celia Mora and Alejandro Uncos for technical support. Drs Alberto Marinconz, Liliana Canini and Néstor Taranto helped with their guidance in the endemic area.

\section{REFERENCES}

Aguilar CM, Rangel EF, Grimaldi Filho G, Momem H 1987. Human, canine and equine leishmanioasis caused by Leishmania braziliensis braziliensis in an endemic area in the State of Rio de Janeiro. Mem Inst Oswaldo Cruz 82: 143.

Campanini AR, Sinagra A, Saravia N, Luna C, Sosa S, Salomón D, Segura E 1993. Caracterización in vitro de aislados de leishmania de pacientes de Salta. Medicina (Bs As) 53(Suppl. 1): 81.
Chiaramonte MG, Frank FM, Furer GM, Taranto NJ, Margni RA, Malchiodi EL 1999. Polymerase chain reaction reveals Trypanosoma cruzi infection suspected by serology in cutaneous and mucocutaneous leishmaniosis patients. Acta Trop 72: 295-308.

Cuba CA, Miles MA, Vexenat A, Barker DC, Mc Mahon Pratt D, Butcher J, Barreto AC, Marsden PD 1985. A focus of mucocutaneous leishmaniasis in Três Braços, Bahia, Brazil: characterization and identification of Leishmania stocks isolated from man and dogs. Tran R Soc Trop Med Hyg 79: 500-507.

Cuba CA, Torno CO, Ledesma O, Visciarelli E, Garcia S, Prat MI, Costamagna R, Barbieri L, Evans DA 1996. Human cutaneous leishmaniasis caused by Leishmania (Viannia) braziliensis in Santiago del Estero, Argentina: identification of parasites by monoclonal antibodies and isoenzymes. Rev Inst Med Trop São Paulo 38: 413-21.

Kirkpatrik CE, Nolan TJ, Farrell JP 1987. Rate of Leishmania-induced skin-lesion development in rodents depends on the site of inoculation. Parasitology 94 : 451-465.

Malchiodi EL, Chiaramonte MG, Taranto NJ, Zwirner NW, Margni RA 1994. Cross-reactivity studies and differential serodiagnosis of human infections caused by Trypanosoma cruzi and Leishmania spp.: the use of immunoblotting and ELISA with a purified antigen (Ag 163B6). Clin Exp Immunol 97: 417-423.

Padilla AM, Marco JD, Diosque P, Malchiodi E, Fernández M, Marinconz A, Basombrío MA 1999. Leishmaniasis tegumentaria canina: evolución de las lesiones y carga parasitaria. Medicina (Bs As) 59 (Suppl. III): 54.

Quinnell RJ, Courtenay O, Dye C 1997. Epidemiology of canine leishmaniasis: transmission rates estimated from a cohort study in Amazon Brazil. Parasitology 115: 143-156.

Reithinger R, Davies CR 1999. Is the domestic dog ( $\mathrm{Ca}$ nis familiaris) a reservoir host of American cutaneous leishmaniasis? A critical review of the current evidence. Am J Trop Med Hyg 61: 530-541.

Sosa Estani S, Campanini A, Sinagra A, Luna C, Peralta M, Contada V, Medina L, Riarte A, Salomón D, Gómez A, Segura E 1998. Características clínicas y diagnóstico de la leishmaniasis mucocutánea en pacientes de un área endémica de Salta. Medicina (Bs As) 58: 685-691.

Sinagra A, Riarte A, Luna C, Campanini A, Segura EL 1997. Leishmania (Viannia) braziliensis: biological behavior in golden hamsters of isolates from Argentine patients. Am J Trop Med Hyg 57: 115-118.

Vexenat JA, Barretto AC, Rosa AC 1986. Experimental infection of Lutzomyia whitmani in dogs infected with Leishmania braziliensis braziliensis. Mem Inst Oswaldo Cruz 81: 125-126.

WHO 1990. Lucha contra la leishmaniasis. Technical Report Series, 793, Geneva, p. 110-128. 